\title{
Density Structure in Two-Component Spin-Orbit-Coupled Dipolar Ultracold Bose Gas
}

\author{
Qiang ZhaO*, Li-Chao Feng, XiaO-Meng Yang and He-En Xu \\ School of Science, North China University of Science and Technology, Tangshan 063210, China
}

Received: 26.08.2021 \& Accepted: 09.11.2021

Doi: $10.12693 /$ APhysPolA.140.427

*e-mail: zhaoqiangac2004@sina.com

\begin{abstract}
In this paper, we consider the ground state properties of a spin-orbit coupled binary Bose-Einstein condensates with dipole-dipole interaction. Our results are obtained by numerically solving the GrossPitaevskii equation in the mean field. It is shown that the density structures are greatly affected by spin-orbit coupled strength and dipole strength. With the increase of spin-orbit coupled strength, we find that a stripe phase and phase separation of two components appear, and the number of stripes increases. In addition, concentric circularity stripes with spatial symmetry appear. We also verify that the phase separation can be broken as the dipole strength increases. However, the stripe phase is still kept in this process. As a result, there are no regions of zero density inside the cloud and the nonfragmented density BEC is exhibited. Also, the critical dipole strength decreases with increasing the SOC strength for the non-fragmented state. This result reflects a competition in which the spin-orbit coupling dominates the dipole-dipole interaction.
\end{abstract}

topics: spin-orbit coupled, dipolar interaction, Bose-Einstein condensates

\section{Introduction}

Spin-orbit coupling (SOC) has long been known and studied in various physical systems. It plays a significant role in semiconductor nanostructures, where spintronics gave rise to many new applications [12]. Recently, the experimental synthesis of SOC for pseudo-spin-half ultracold atoms has made a great breakthrough [3, 4]. Such systems have been realized in Bose [5] as well as in Fermi gases [6]. Their unique properties and fundamental and application-related prospects have inspired intense theoretical studies [7-11]. Furthermore, these systems emulate the Rashba and Dresselhaus SOC for electrons in solids which has a deep influence on many condensed-matter phenomena [12]. For review properties of SOC in Bose-Einstein condensates (BECs) see [13] and references therein.

On the other hand, dipolar BECs have been created with ${ }^{52} \mathrm{Cr}$ [14], ${ }^{164} \mathrm{Dy}[15]$ and ${ }^{168} \mathrm{Er}$ [16] atoms. These atoms have large magnetic moments induced by dipole-dipole interactions (DDI) which are longranged and anisotropic. The interplay of the DDI and a short-range contact interaction makes particles intriguing from both the experimental and the theoretical point of view [17, 18], for example, Rosensweig instability [19] and roton quasiparticles [20]. In particular, the emergence of quantumstabilised droplets further inspires much research including dipolar quantum gases in a rotating trap [21-23], the formation of the droplets [24, 25], elementary excitations [26, 27], the effect of threebody interactions [28] and the self-bounded nature of the droplets [29].

Recently, there has been an intense focus on the topic of binary SOC BECs with DDI. Chiquillo [30] studied the formation of stable bright solitons in quasi-one-dimensional spin-orbit- and Rabicoupled dipolar BECs, and a plane-wave soliton and a stripe soliton were found [30]. The creation of a long-lived SOC gas of quantum degenerate atoms using dysprosium atoms was reported in [31]. In addition, the authors showed that the degenerate dispersion minimum due to the SOC and DDI can stabilize many quantum crystallines and a quasicrystalline ground state [32]. Coupling of the bosons to fermionic species can further stabilize these phases. The Fermi liquid states of the ultracold magnetic dipolar Fermi gases in the two-components including both thermodynamic instabilities and collective excitations have also been studied [33].

In this paper, we study the ground state of SOC binary BECs with DDI. Numerical results are obtained by evolving the imaginary time GrossPitaevskii (GP) equation. We find that the density structures are greatly affected by the SOC strength and the dipole strength. With the increase of the SOC strength, it is shown that the stripe phase and phase separation occur and the stripe number increases. In addition, the concentric circularity stripe 
occurs and the spatial symmetry is embodied. Moreover, we show that the phase separation can be broken while the stripe phase is still kept with increasing dipole strength. As a result, there is nearly no point of zero density in space, i.e., non-fragmented density BEC is exhibited. Also, the critical dipole strength decreases with increasing SOC strength. Our results reflect the fact that the SOC dominates the DDI for the formation of the non-fragmented density BEC.

The paper is structured as follows. In Sect. 2, we give the theoretical model and briefly introduce the numerical method. The effects of the SOC strength and the dipole strength on the ground state density structure are discussed in Sect. 3. Finally, Sect. 4 summarizes our concluding remarks and gives an outlook for future research.

\section{Theoretical model}

We consider a spin-orbit coupled dipolar BEC consisting of $N$ atoms with spin- $1 / 2$ and trapped in the harmonic potential. The Hamiltonian of the system can be well described by the following coupled GP equations [34]

$$
\begin{aligned}
& \mathrm{i} \hbar \frac{\partial \psi_{1}}{\partial t}=\left(-\frac{\hbar^{2}}{2 m} \nabla^{2}+V(\boldsymbol{r})+g_{11}\left|\psi_{1}\right|^{2}+g_{12}\left|\psi_{2}\right|^{2}+g_{d} \int \mathrm{d} \boldsymbol{r}^{\prime} v_{\mathrm{dip}}\left(\boldsymbol{r}^{\prime}-\boldsymbol{r}\right)\left|\psi_{1}\left(\boldsymbol{r}^{\prime}, t\right)\right|^{2}\right) \psi_{1}-\lambda\left(\mathrm{i} \partial_{x}+\partial_{y}\right) \psi_{2} \\
& \mathrm{i} \hbar \frac{\partial \psi_{2}}{\partial t}=\left(-\frac{\hbar^{2}}{2 m} \nabla^{2}+V(\boldsymbol{r})+g_{21}\left|\psi_{1}\right|^{2}+g_{22}\left|\psi_{2}\right|^{2}\right) \psi_{2}-\lambda\left(\mathrm{i} \partial_{x}-\partial_{y}\right) \psi_{1}
\end{aligned}
$$

where $\psi_{j}=\psi_{j}(\boldsymbol{r}, t)$ is the $j$-th wave function of a two-component system $\left(j=1,2, \boldsymbol{r}=(x, y) \in R^{2}\right)$. Now, $m$ is the atomic mass and $\lambda$ is the SOC strength which carries the unit of velocity. The coefficients $g_{11}, g_{22}$ and $g_{12}, g_{21}$, respectively, are the strengths of intra- and intercomponent coupling interactions, i.e., $g_{i i}=\frac{4 \pi \hbar^{2} a_{i i}}{m}$ and $g_{i j}=\frac{2 \pi \hbar^{2} a_{i j}}{m}$. Here, $a_{i i}$ and $a_{i j}$ denote the $s$-wave scattering lengths between intra- and intercomponent atoms. In turn, $g_{d d}=\mu_{0} \mu^{2} /(4 \pi)$ is the DDI coupling constant, where $\mu_{0}$ is the magnetic vacuum permeability and $\mu$ is the dipole moment. For simplicity, we set $g_{11}=g_{22}$ and $g_{12}=g_{21}$. The external trapping potential is $V(\boldsymbol{r})=\frac{1}{2} m \omega^{2}\left(x^{2}+y^{2}\right), \lambda=\frac{\omega_{z}}{\omega}$ is the aspect ratio, $\omega$ and $\omega_{z}$ are trap frequencies in the $x y$-plane and the $z$-axis. The DDI is described by the potential $v_{\text {dip }}\left(\boldsymbol{r}-\boldsymbol{r}^{\prime}\right)=\frac{1-3 \cos ^{2}(\alpha)}{\left|\boldsymbol{r}-\boldsymbol{r}^{\prime}\right|^{3}}$, where $\boldsymbol{r}-\boldsymbol{r}^{\prime}$ is the distance between the dipoles, and $\alpha$ is the angle between the polarization axis $\boldsymbol{n}$ and the relative position of two atoms, i.e, $\cos (\alpha)=\boldsymbol{n} \cdot \boldsymbol{r} /|\boldsymbol{r}|$.

By introducing the dimensionless variables $\tilde{t}=\omega t, \quad \tilde{r}=r / a_{0}, \quad \tilde{\psi}=\psi a_{0}^{3 / 2} / \sqrt{N}$ with $a_{0}=$ $\sqrt{\hbar /(m \omega)}$, and $\tilde{\kappa}=\kappa \sqrt{\frac{m}{\hbar \omega}}$, we can get the dimensionless GP equation in the following form after omitting the tildes,

$$
\begin{aligned}
& \mathrm{i} \frac{\partial \psi_{1}}{\partial t}=\left(-\frac{1}{2} \nabla^{2}+\frac{x^{2}+y^{2}}{2}+\beta_{11}\left|\psi_{1}\right|^{2}+\beta_{12}\left|\psi_{2}\right|^{2}+\beta_{d} \Phi_{\mathrm{D}}\right) \psi_{1}-\kappa\left(\mathrm{i} \partial_{x}+\partial_{y}\right) \psi_{2}, \\
& \mathrm{i} \frac{\partial \psi_{2}}{\partial t}=\left(-\frac{1}{2} \nabla^{2}+\frac{x^{2}+y^{2}}{2}+\beta_{21}\left|\psi_{1}\right|^{2}+\beta_{22}\left|\psi_{2}\right|^{2}\right) \psi_{2}-\kappa\left(\mathrm{i} \partial_{x}-\partial_{y}\right) \psi_{1}, \\
& \Phi_{\mathrm{D}}=\left[n_{3}^{2}\left(\frac{\partial^{2}}{\partial x^{2}}+\frac{\partial^{2}}{\partial y^{2}}\right)-\partial_{\boldsymbol{n}_{\perp}} \boldsymbol{n}_{\perp}\right] \int \mathrm{d} \boldsymbol{r}^{\prime} U_{\mathrm{D}}\left(\boldsymbol{r}-\boldsymbol{r}^{\prime}\right)\left|\psi_{1}\left(\boldsymbol{r}^{\prime}, t\right)\right|^{2},
\end{aligned}
$$

where

$$
\beta_{i i}=\frac{4 \pi a_{i i} N \sqrt{\lambda}}{\sqrt{2 \pi} a_{0}}, \quad \beta_{d}=\frac{\mu_{0} \mu^{2} m N \sqrt{\lambda}}{3 \sqrt{2 \pi} \hbar^{2} a_{0}}
$$

and $i=1,2$. The term $\Phi_{\mathrm{D}}$ is the dipole integral with $\partial_{\boldsymbol{n}_{\perp}}=n_{1} \partial_{x}+n_{2} \partial_{y}$ and $\partial_{\boldsymbol{n}_{\perp} \boldsymbol{n}_{\perp}}=\partial_{\boldsymbol{n}_{\perp}}\left(\partial_{\boldsymbol{n}_{\perp}}\right)$. The kernel part in $(5), U_{\mathrm{D}}$, is radially symmetric and is given as $U_{\mathrm{D}}(r)=\left(\frac{\lambda}{2 \pi}\right)^{\frac{3}{2}} \mathrm{e}^{r^{2} / 4} K_{0}\left(\lambda r^{2} / 4\right)$, where $K_{\nu}$ denotes a modified Bessel function of the second kind $(\nu$ real $)$ and $r^{2}=\left(x-x^{\prime}\right)^{2}+\left(y-y^{\prime}\right)^{2}$.
Numerically, we compute (3)-(5) by the convolution theorem with the fast Fourier transform [36]. Similarly, kinetic energy can be calculated with high accuracy using the Fourier transform of the wave function to momentum space [37]. Finally, the ground states can be obtained by using the imaginary time evolution method [38], where one makes a simple replacement it $\rightarrow \tau$. We solve (3)-(5) on a $256 \times 256$ square grid. The computational domain is chosen as a square $[-5,5] \times[-5,5]$ and the time step is $\Delta t=0.001$. 


\section{Results and discussion}

In this section, we report numerical results for the ground state of SOC binary BECs with DDI. The parameters of intercomponent interaction and intracomponent interaction are $\beta_{11}=\beta_{22}=1000$ and $\beta_{12}=\beta_{21}=1100$, respectively. With this choice, it is easy to observe the phase of the stripe. In addition, we study the case of the isotropic repulsive interaction $\alpha=0$ when the aspect ratio is $\lambda=20$. Throughout this article, the tunable parameters are the SOC strength $\kappa$ and the dipole strength $\beta_{d}$. In what follows we first perform numerical calculations to investigate the effect of SOC on the density structure, and then move on to the effect of the dipole strength on the system. As a possibility of experimental observation, we propose an experimental scheme using these control parameters.

In Fig. 1, we show the density profiles of the system at fixed dipole strength $\beta_{d}=30$. Different SOC strengths $\kappa=1.0,2.0,3.0,4.0$, and 5.0 are considered. The rows in Fig. 1, top and bottom, correspond to the density of the component $\psi_{1}$ and $\psi_{2}$, respectively. The phase distributions $\theta_{j}(x, y)=\arctan \left(\frac{\operatorname{Im}\left[\psi_{j}(x, y)\right]}{\operatorname{Re}\left[\psi_{j}(x, y)\right]}\right)$ are shown in Fig. 2. To the best of our knowledge, the stripe phase can occur in simply connected condensates $[39,40]$ when SOC is included, and the necklace-like petal state is formed in the toroidal trap [41, 42]. As shown in Fig. 1, the density distribution of each component is sensitive to the SOC strength.

When the SOC strength is $\kappa=1.0$, phase separation of two components appears where the minima of the $\psi_{1}$ component correspond to the maxima of the $\psi_{2}$ component. The direction of stripes is along the $x$-axis. By simply counting, the number of stripes is 4 and 3 in the $\psi_{1}$ and $\psi_{2}$ component, respectively. Since the SOC strength in this case is relatively weak, therefore the SOC effect on the density structure is relatively small.

As the SOC strength reaches 2.0, the state of the system changes. The number of stripes increases and the stripe orientation is aligned almost along the $y$-axis. For a larger value $\kappa=3.0$, the number of stripes becomes 11 and the direction of stripes varies again. When we continue to increase the SOC strength value to 4.0 , it becomes clear that there is no essential change in the structure of the stripes. However, the direction of their alignment becomes consistent with the result for $\kappa=1.0$. As the SOC is further increased to $\kappa=5.0$, the stripes have concentric circularity and thus present spatial symmetry which is obviously different from the previous cases. Concurrently, the property of the phase separation is kept and the number of annular stripes is still 11 in each component. This was also noted in [43] where the ground-state properties of Rashba SOC pseudo-spin-1/2 BECs in a rotating two-dimensional toroidal trap are discussed. The stripe phase is obtained with only the $x$ direction SOC and thus the fringes are naturally arranged

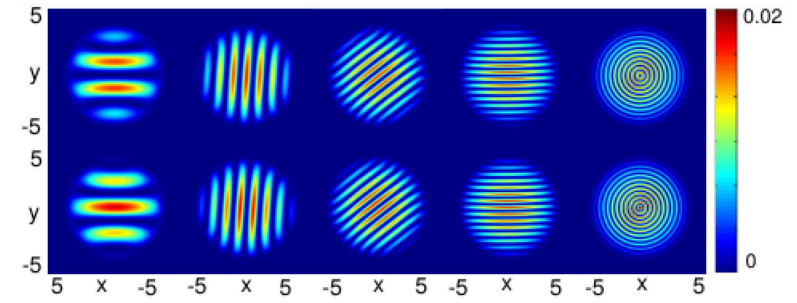

Fig. 1. The ground state density profiles for two components. From top to bottom, the row represents $\left|\psi_{1}\right|^{2}$ and $\left|\psi_{2}\right|^{2}$. From left to right, the SOC strength $\kappa$ is $1.0,2.0,3.0,4.0$ and 5.0 , respectively. The dipole strength is fixed with $\beta_{d}=30$.

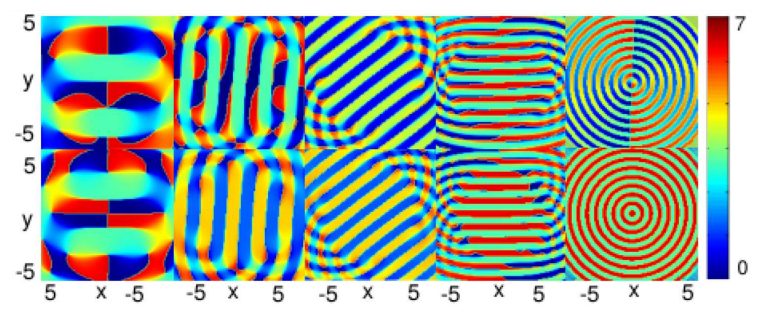

Fig. 2. The phase profiles for two components. From top to bottom, the row represents $\left|\psi_{1}\right|^{2}$ and $\left|\psi_{2}\right|^{2}$. From left to right, the SOC strength $\kappa$ is $1.0,2.0,3.0,4.0$ and 5.0, respectively. The dipole strength is fixed with $\beta_{d}=30$.

along the $x$ direction, as shown in Fig. 2 in [43]. The stripe phase in our manuscript is based on the isotropy of SOC in $x$ and $y$ direction, and the BECs are confined in the harmonic trap with DDI. The heliciform stripe structures and annular stripes are created with increasing $\beta_{12}$ in [43] and in our study - with the increase of SOC strength $\kappa$.

It is important to emphasize that the increasing SOC strength is more advantageous to break the symmetry of spatial translation in rotating BECs such as Fig. 7 in [43]. Nevertheless, we exhibit the appearance of symmetry in this manuscript. The reason for this difference is likely the confinement of a toroidal trap, which gives rise to the heliciform structure. Also, the physics of the annular stripe is the competition between the SOC and DDI.

It is also interesting to investigate the effect of dipole strength on the density profile of the system. Figure 3 exhibits the density patterns for the following values $\beta_{d}=10.0,50.0,97.0,98.0$, and 99.0. It is evident that the stripe phase and phase separation is kept until $\beta_{d}=97.0$. For the weak increases, SOC strength $\beta_{d}=98.0$ even to 99.0 , the stripe is still seen but the nature of the phase separation is lost. This point is verified from the phase distribution, as shown in Fig. 4. These facts confirm that increasing the dipole strength is helpful in breaking the phase separation. As a result, there is nearly no point of zero density in space, namely non-fragmented density BEC forms. The reason for this state is that the number of stripes becomes denser and denser. 


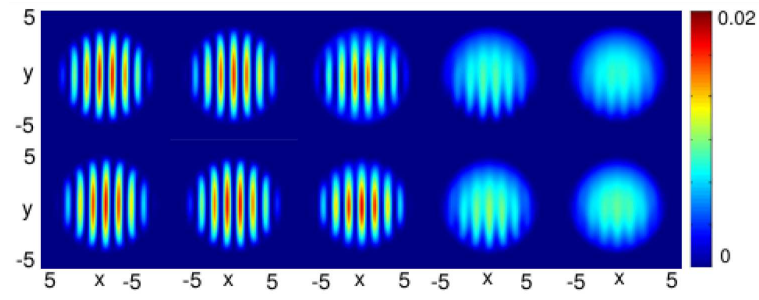

Fig. 3. The ground state density profiles for two components. From top to bottom, the row represents $\left|\psi_{1}\right|^{2}$ and $\left|\psi_{2}\right|^{2}$. From left to right, the dipole strength $\beta_{d}$ is $10.0,50.0,97.0,98.0$ and 99.0, respectively. The SOC strength is fixed with $\kappa=2.0$.

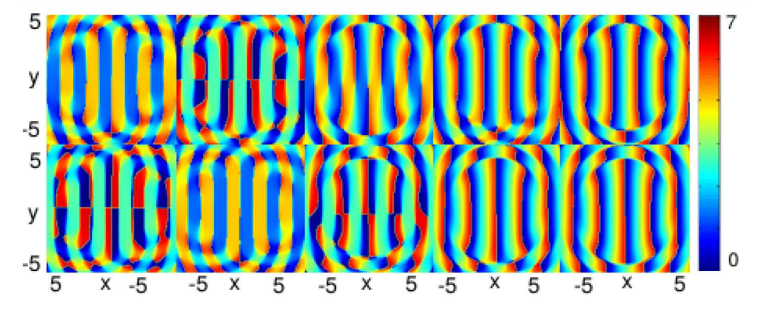

Fig. 4. The phase profiles for two components. From top to bottom, the row represents $\left|\psi_{1}\right|^{2}$ and $\left|\psi_{2}\right|^{2}$. From left to right, the dipole strength $\beta_{d}$ is 10.0, 50.0, 97.0, 98.0 and 99.0, respectively. The SOC strength is fixed with $\kappa=2.0$.

One of the most crucial issues in the SOC BECs is the generation of the stripe phase. This point has been discussed in more detail by Zhai in [8], where the ground states of spin- $1 / 2$ and spin- 1 BECs with Rashba SOC are obtained. To further explore the effect of DDI on the formation of the nonfragmented density BEC, we compute the critical dipole strength $\beta_{d c}$ as a function of SOC strength $\kappa$, as shown in Fig. 5 . It is obvious that $\beta_{d c}$ decreases monotonically with $\kappa$. These observations can be explained as follows. Increasing of the SOC strength makes the stripe grow closer. This result is helpful in forming the non-fragmented density BEC. Meanwhile, under the present circumstances, we consider the purely repulsive and isotropic DDI. Increasing of dipole strength makes the condensates expand and the dipolar atoms have high enough kinetic energies to occupy the cloud. As a consequence, the non-fragmented density BEC is easily created. Therefore, increasing the SOC strength and increasing the dipole strength plays the same role in the formation of non-fragmented density BEC. In addition, we can see that the bigger the SOC strength, the smaller dipole strength is needed to reach the non-fragmented state. This reflects the fact that the SOC dominates the DDIs for the current considered system, which is a specific reflection of the competition relationship between the SOC and DDI.

Finally, the possibility to observe these phenomena in real experiments is briefly discussed. In the area of ultracold atom physics, the two-component

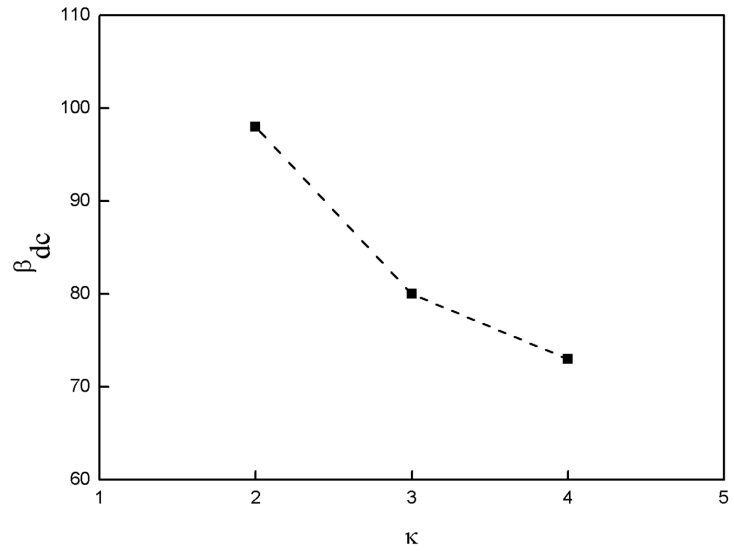

Fig. 5. Critical dipole strength $\beta_{d c}$ as a function of SOC strength $\kappa$ when the system reaches the nonfragmented density BEC.

BECs can be created by adjusting two ground state hyperfine manifolds of atomics with spin projections $m_{J}=-J$ and $m_{J}=0$. The SOC with BECs is realized in a cold atom system by engineering atomlight interactions and its strength is set by the wavelength of a pair of Raman lasers and intersection angle [5]. Also, the polarization angle $\alpha$ can be flexibly adjusted by an external polarized field. In addition, the dipole strength and the inter- and intracomponent interactions can be tuned by means of rotating the orienting field [44] and Feshbach resonance [45], respectively. Therefore, the current studied phenomena in this paper can be verified and further explored in cold atom physics experiment.

\section{Summary}

We systematically investigated the ground state properties of the SOC two-component BECs with DDI which was trapped in a harmonic trap. Our results demonstrate that the density structures are strongly influenced by the SOC strength and the dipole strength. With increasing the SOC strength, the stripe phase follows and the phase separation of two components occurs, and the number of stripes increases. Furthermore, the concentric circularity stripe appears which shows spatial symmetry at the maximum considered SOC strength. This point differs from the previous study. In addition, we find that increasing of dipole strength can destroy the phase separation. Nevertheless, the stripe phase is still kept. As a result, non-fragmented density BEC is exhibited. In addition, the critical dipole strength decreases with increasing of the SOC strength. This result reflects the fact that the SOC dominates the DDI for the formation of non-fragmented density BEC.

We indicate that there are many open questions for future study in this subject. From a theoretical perspective, it would also be quite intriguing to explore the effect of the anisotropic of DDI on the density structure in the current system. For instance, 
$\alpha$ changes from zero to $\pi / 2$. In this case, the interaction is repulsive along the $y$ axis and attractive along the $x$ axis. We note that the anisotropic displays a rich physical picture [46]. This is a feature worth further elucidating in future theoretical analyses. Simultaneously, we also notice that the present model could be easily extended to other situations, for example, to include a rotation, the anisotropic of SOC in different axes, the Bogoliubov excitation spectrum and mass imbalance. These ideas could be explored and provide valuable information for understanding the new physics.

\section{Acknowledgments}

This work was supported by the Ph.D. Start-up Fund (Grant No. BS2017096) of North China University of Science and Technology.

\section{References}

[1] M.Z. Hasan, C.L. Kane, Rev. Mod. Phys. 82, 3045 (2010).

[2] X.L. Qi, S.C. Zhang, Rev. Mod. Phys. 83, 1057 (2011).

[3] Y.J. Lin, K. Jiménez-García, I.B. Spielman, Nature 471, 83 (2011).

[4] J.Y. Zhang, S.C. Ji, Z. Chen et al., Phys. Rev. Lett. 109, 115301 (2012).

[5] Y.J. Lin, R.L. Compton, K. JiménezGarcía, J.V. Porto, I.B. Spielman, Nature 462, 628 (2009).

[6] P. Wang, Z.Q. Yu, Z. Fu, J. Miao, L. Huang, S. Chai, H. Zhai, J. Zhang, Phys. Rev. Lett. 109, 095301 (2012).

[7] I.B. Spielman, Phys. Rev. A 79, 063613 (2009).

[8] C. Wang, C. Gao, C.M. Jian, H. Zhai, Phys. Rev. Lett. 105160403 (2010).

[9] T.L. Ho, S. Zhang, Phys. Rev. Lett. 107, 150403 (2011).

[10] X. Yan, F. Yuan, Q. Gu, Solid State Commun. 279, 1 (2018).

[11] J.X. Zhao, Q. Gu, Sci. Rep. 8, 892 (2018).

[12] D. Xiao, M.C. Chang, Q. Niu, Rev. Mod. Phys. 82, 1959 (2010).

[13] H. Zhai, Rep. Progr. Phys. 78, 026001 (2015).

[14] A. Griesmaier, J. Werner, S. Hensler, J. Stuhler, T. Pfau, Phys. Rev. Lett. 94, 160401 (2005).

[15] M. Lu, S.H. Youn, B.L. Lev, Phys. Rev. Lett. 104, 063001 (2010).

[16] K. Aikawa, A. Frisch, M. Mark, S. Baier, A. Rietzler, R. Grimm, F. Ferlaino, Phys. Rev. Lett. 108, 210401 (2012).

[17] M.A. Baranov, M. Dalmonte, G. Pupillo, P. Zoller, Chem. Rev. 112, 5012 (2012).
[18] T. Lahaye, C. Menotti, L. Santos, M. Lewenstein, T. Pfau, Rep. Prog. Phys. 72, 126401 (2009).

[19] H. Kadau, M. Schmitt, M. Wenzel, C. Wink, T. Maier, I. Ferrier-Barbut, T. Pfau, Nature 530, 194 (2016).

[20] L. Chomaz, R.M.W. van Bijnen, D. Petter, G. Faraoni, S. Baier, J. Hendrik Becher, M.J. Mark, F. Wachtler, L. Santos, F. Ferlaino, Nat. Phys. 14, 442 (2018).

[21] A. Gallemí, S.M. Roccuzzo, S. Stringari, A. Recati, Phys. Rev. A 102, 023322 (2020).

[22] M. Nilsson Tengstrand, D. Boholm, R. Sachdeva, J. Bengtsson, S.M. Reimann, Phys. Rev. A 103, 013313 (2021).

[23] F. Ancilotto, M. Barranco, M. Pi, L. Reatto, Phys. Rev. A 103, 033314 (2021).

[24] K.T. Xi, H. Saito, Phys. Rev. A 93, 011604 (2016).

[25] D. Baillie, R.M. Wilson, R.N. Bisset, P.B. Blakie, Phys. Rev. A 94, 021602 (2016).

[26] R.N. Bisset, R.M. Wilson, D. Baillie, P.B. Blakie, Phys. Rev. A 94, 033619 (2016).

[27] D. Baillie, R.M. Wilson, P.B. Blakie, Phys. Rev. Lett. 119, 255302 (2017).

[28] P.B. Blakie, Phys. Rev. A 93, 033644 (2016).

[29] D. Baillie, R.M. Wilson, R.N. Bisset, P.B. Blakie, Phys. Rev. A 94, 021602 (2016).

[30] E. Chiquillo, Phys. Rev. A 97, 013614 (2018).

[31] N.Q. Burdick, Y.J. Tang, B.L. Lev, Phys. Rev. X 6, 031022 (2016).

[32] S. Gopalakrishnan, I. Martin, E.A. Demler, Phys. Rev. Lett. 111, 185304 (2013).

[33] Y. Li, C.J. Wu, Phys. Rev. B 85, 205126 (2012).

[34] Q. Zhao, H.J. Bi, Int. J. Theor. Phys. 60, 1603 (2021).

[35] S. Gautam, J. Phys. B 47, 165301 (2014).

[36] Y.Y. Cai, M. Rosenkranz, Z. Lei, W.Z. Bao, Phys. Rev. A 82, 043623 (2010).

[37] W.Z. Bao, D. Jaksch, P.A. Markowich, J. Comput. Phys. 187, 318 (2003).

[38] W.Z. Bao, I.L. Chern, F.Y. Lim, J. Comput. Phys. 219, 836 (2006).

[39] J.R. Li, J. Lee, W. Huang, S. Burchesky, B. Shteynas, F.C. Top, A.O. Jamison, W. Ketterle, Nature (London) 543, 91 (2017). 
[40] X.F. Zhang, M. Kato, W. Han, S.G. Zhang, H. Saito, Phys. Rev. A 95, 033620 (2017).

[41] A.C. White, Y. Zhang, T. Busch, Phys. Rev. A 95, 041604 (2017).

[42] J.G. Wang, L.L. Xu, S.J. Yang, Phys. Rev. A 96, 033629 (2017).

[43] H. Wang, L.H. Wen, H. Yang, C.X. Shi, J.H. Li, J. Phys. B 50, 155301 (2017).
[44] S. Giovanazzi, A. Görlitz, T. Pfau, Phys. Rev. Lett. 89, 130401 (2002).

[45] C. Chin, R. Grimm, P. Julienne, E. Tiesinga, Rev. Mod. Phys. 82, 1225 (2010).

[46] Q. Zhao, J. Low. Temp. Phys. 204, 1 (2021). 